\title{
Feature Extraction of the Wear Label of Carpets by Using a Novel 3D Scanner
}

\author{
S. A. Orjuela ${ }^{a}$, E. Vansteenkiste ${ }^{a}$, F. Rooms ${ }^{a}$, S. De Meulemeester ${ }^{b}$, R. de $\operatorname{Keyser}^{c}$ and \\ W.Philips ${ }^{a}$ \\ ${ }^{a}$ Department of Telecommunications and Information Processing (TELIN), IPI, IBBT, Ghent \\ University, Sint-Pietersnieuwstraat 41, B-9000 Gent, Belgium; \\ ${ }^{b}$ Department of Textile, Ghent University, Belgium; \\ ${ }^{c}$ Department of Electrical energy, Systems and Automation, Ghent University, Belgium
}

\begin{abstract}
In this paper we present a novel 3D scanner to capture the texture characteristics of worn carpets into images of the depth. We first compare our proposed scanner to a Metris scanner previously attempted for this application. Then, we scan the surface of samples from the standard EN1471 using our proposed scanner. We found that our proposed scanner offers additional benefits because it has been specifically designed for carpets, performing faster, cheaper, better and also a lot more suitable for darker carpets. The results of this approach give optimistic expectations in the automation of the label assessment dealing with multiple types of carpets.
\end{abstract}

Keywords: Image processing, Local Binary Patterns, Wear label, Textile floor covering inspection

\section{INTRODUCTION}

In the textile floor covering industry, manufacturers certify their products for end-use applications through visual assessment. This is performed by human experts. Minimally three experts grade worn samples previously subjected to accelerated mechanical wear to simulate traffic exposure. ${ }^{2}$ The experts compare the worn samples to rated reference samples. Despite the best comparison is performed using physical sample references, ${ }^{1}$ a normalization committee decided to propose certified photographs by the Carpet and Rug Institute (CRI) ${ }^{3}$ because physical features change over time.

For years, textile floor coverings in Europe have been compared using the human experts system. However, manufacturers require a more objective assessment because the human assessment is somewhat subjective. With this objective in mind, research in automated rating has progressed, specifically on image processing techniques extracting texture parameters from digital color images. ${ }^{4-11}$ Particularly; the Department of Textiles in Ghent University has been conducting research in automated wear label rating for the last 15 years. In 1996, an agreement with TFI (The Deutsche Teppich-Forschungsinstitut ${ }^{12}$ ) yield to an automatic assessment for cut pile textile floor coverings using image analysis and neural networks. However, manufacturers at that time considered a $90 \%$ of correct assessment achieved in the classification insufficient for practical purposes. Afterwards in 2000, in collaboration with the companies Desso, Lano and Balta, the required accuracy of the classification was satisfying for some types of carpets. However, the results were not considered generic enough for practical applications because each type of carpets required different algorithms. ${ }^{14}$

During his PHd, from 2004 to 2006, Willem Waegeman used information of the surface of the textile floor coverings obtained from a 3D laser scanner; model Metris LC50, instead of the traditional color images. ${ }^{13}$ The 3D laser was used in search for adequately capture the three dimensional structure of the carpet evaluated by the experts. This type of scanner is mainly used for high speed digitization of relatively large objects. Waegeman obtained correlations up to 0.77 between features and wear labels and also obtained a correct

Further author information: (Send correspondence to S. A. Orjuela) S. A. Orjuela is supported by a grant number 20070207 of the 'LASPAU' Academic and Professional Programs for the Americas in agreement with COLCIENCIAS and Universidad Antonio Nariño, Colombia: E-mail: seraleov@telin.ugent.be, Telephone: +32 92643415 
assessment of $76 \%$ if an error in class of 0.5 is allowed and of $97 \%$ if a serious error in class of 1.0 is allowed. Waegeman's results were the basis of the latest project named COMPAS (COMPuter-based ASsessment of Aspect Change in Carpets due to Wear), starting from end of 2006 at Ghent University. This project attempts to find a universal classification system for carpets using laser and/or digital image data. ${ }^{14}$

The last step of the COMPAS project has been conducted in collaboration with both the Department of TELecommunications and INformation processing (TELIN) and the department of Electrical energy, Systems and Automation (EeSA), both also from Ghent University.

Under this collaboration, we applied image processing on the data acquired from the LC50 laser scanner. The resulting scanned data contains the followings characteristics that are not suitable for image processing: i) data points are spread in a nonstructural grid, ii) the number of the acquired points is dependent of the color of the carpet, and iii) the use of block of woods to hold the carpet limits the surface that can be scanned. Therefore, a transformation from 3D data to a 2D grid has to be performed. We were able to correctly classify over $95 \%$ of ten types of textile floor coverings. ${ }^{15}$ For this, we extracted features using the coocurrence matrix and classified using a Gaussian Kernel on Support vector machines. Although the percentage of classification was high, the extracted features were not ranked according to the wear labels as should be expected.

In the current approach we present an alternative 3D scanner based on structured light. This new 3D laser scanning system is less dependent on the ilumination conditions and color of the carpet and obtains data points on a structured grid instead of sparse points. Therefore, the scanned images are more appropriate for image analysis. The new system is also more than five times cheaper, scans more than seven times faster and is specifically designed for scanning carpets instead of 3D objects. We first compare the performance of the new 3D laser scanner to the LC50 laser scanner using three types of loop pile texture floor coverings. Afterwards, we use our 3D scanner to acquire eight types of references. The wear labels of these references are estimated using LBP statistics.

The paper is organized as follows. In Section 2 we describe first the current practice of visual inspection of the degree of wear in carpets followed by the details of our proposed scanner and the carpet batch to be analyzed. In Section 3 we present the method to compare the performance of both scanners. In Section 4 we report the results of the comparison as well as the results using the scanner on the test samples. Finally, in Sections 5 and 6 findings are discussed and conclusions are drawn.

\section{MATERIALS}

The Carpet Appearance Retention Rating has been designed according to the ASTM D-6119 standard. ${ }^{16}$ It consists of a set of scales, each containing 8 pairs of digital images representing a comparison between the original appearance of the carpet and a worn version. The images of the original appearance are rated 5 while the images of the worn textile floor coverings are rated from 1 to 4.5 in steps of 0.5 , known as wear labels. These ratings reflect the expected appearance change from the original in a given traffic area during the first year after installation [1] under increasing wear conditions. Rating 1 represents severe wear whereas rating 4.5 indicates little change in appearance.

To predict the expected appearance change of a given textile floor covering sample, it is first subjected to wear on the Hexapod or Vetermann device accordance with the ASTM Standards. The measurements of the sample are dependent on the method used: $450 \times 200 \mathrm{~mm}^{2}$ for the Hexapod and $570 \times 265 \mathrm{~mm}^{2}$ for the Vetterman device. As a result, the sample contains both a worn region in the middle and the original texture at both sides. Afterwards, at least three experts visually evaluate changes in the worn region, by comparing both to the unworn parts of the carpet and to a corresponding Carpet Appearance Retention Rating scale. Consequently with the aim of ht COMPAS project, we propose replacing the expert assessment by an automated method based on image analysis to estimate these gradual changes.

Taking into account that 1) the best comparison is performed using physical sample references as well as 2) the good results obtained using the Metris LC50 laser scanner (despite this laser was not appropriate for image analysis), we propose a 3D laser scanner specifically designed for scanning carpets. This scanner acquires the shape of the surface of the textile floor covering based on the structured light method. In this method a camera captures the depth information of an object by projecting a laser line on the object as shown in Figure 1. 


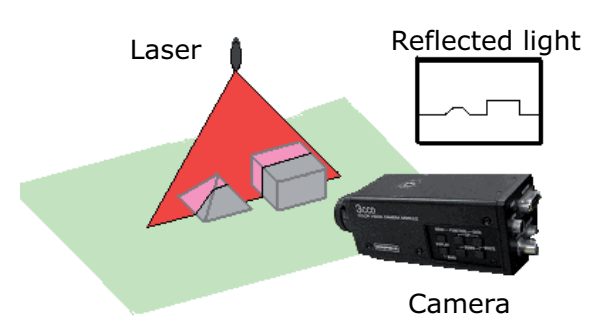

Figure 1: Structured light method to measure depth in 3D shapes based on triangulation.

\subsection{D Scanner based in structure light.}

In our design, we use a camera to acquire the reflection of a line laser on the surface of the textile floor covering (See Figure 2 (a)). Then, the depth information is obtained using a gradient operator and is saved into an array. Moving the surface and saving the laser reflection on a video will result in one array per frame (See Figure 2 (b)). The arrays are added sequentially to build a 2D representation of the depth information, which can be used as an image (See Figure 2 (c)). The final prototype is shown in Figure 2 (d). It consists of:

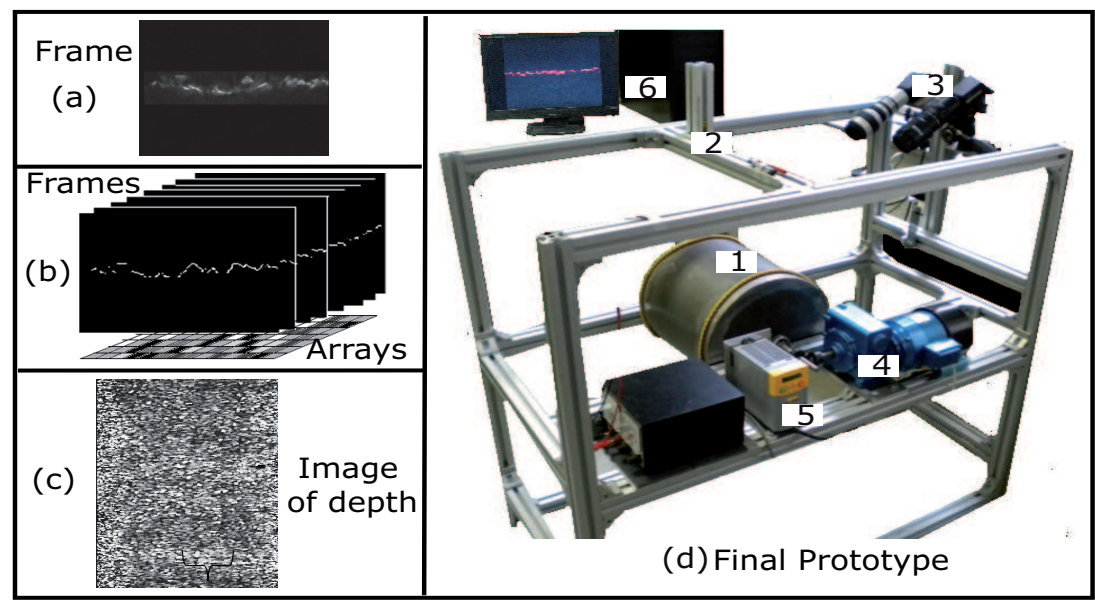

Figure 2: Final prototype.

1. An inox-drum with a diameter of $25 \mathrm{~cm}$ to fix the samples such that they can be scanned correctly. A drum was chosen because bending of the carpet allows for a better exposure to the laser light and hence allows better information of the profile of each row of tufts.

2. Lighting consists of a laser line generator; model LDM-4, with a fan angle of $60^{\circ}$ located $20 \mathrm{~cm}$ above the drum. This laser produces a high quality uniform line of $20 \mathrm{~cm}$ on the surface of the drum with thickness of $0.5 \mathrm{~cm}$. For an appropriated acquisition of the sample surface, the brightness of the line must be uniform across its entire length. Therefore, we selected the uniform intensity laser line generator because it gives significant advantages over standard line generator with Gaussian lines.

3. 2 cameras model JAI CV-M50 with resolution of $720 \times 576$ pixels. Each camera has a lens model $6 \mathrm{X}$ Zoom C-mount. The lenses are distanced $30 \mathrm{~cm}$ from the line on the surface, thus, each camera captures a length of $10 \mathrm{~cm}$ of the surface.

4. A motor model Parvalux 400-753 with gear box to control the distance between captured lines. During this experiment we use the minimum speed which allow us to capture frames spaced $0.24 \mathrm{~mm}$.

5. An AC Inverter Drive Speed Controller with model Parker SSD Drives 650. 
6. A PC to save the recorded images and in which the processing software is integrated.

To compare the performance of the scanners, we scanned samples of three types of loop pile surface of texture floor coverings with light colors, using both our 3D scanner and the Metris LC50 laser scanner. Each type contains six samples, one original, named $\mathrm{O}$, and other five which have been previously subjected to wear using $5,10,15,20$ and 25 thousand revolutions in the vetterman drum. We call these samples the test samples. We defined the worn test samples as $t$, with $t=1$ to 6 corresponding to the number of thousands of revolutions, with $T=6$ the number of different revolutions.

The reason why only three types were compared was because the lease agreement with the owner of the scanner ended and a large number of dark (not necessarily black) textile floor covering samples with the old Metris scanner just resulted in very few and sparse data points.

Afterwards, we scan using our proposed 3D scanner, eight types of textile floor covering references provided from the standard EN1471, ${ }^{17}$ called reference fatigued specimens. These references contain samples from loop, cut/loop, woven cut pile, frise and shaggy surfaces of texture floor coverings including samples with dark colors. Each reference set contains nine rated samples increasing half step from 1 to 5 . We refer to these samples as the reference samples and their ratings as the reference labels, defined as $l$, with $l=1$ to 9 .

\section{METHODS}

To scan using our proposed 3D scanner, the test samples are first vacuumed cleaned. Then, each test sample is held at a time to the drum with elastic bands. We turn the drum during $50 \mathrm{~s}$ capturing 1250 frames per sample. This corresponds to a length of $30 \mathrm{~cm}$ of the surface of the sample. The lens of each camera is calibrated to cover a width of $8.64 \mathrm{~cm}$ on both right and left sides of the sample. We compute arrays of 720 values per stripe, thus, we represent the depth information in cells with size of $0.24 \times 0.12 \mathrm{~mm}^{2}$. Each of these cells corresponds to a pixel of an image where the values of the depth are converted into a rank from 1 to 256 values. Therefore, the depth of the surface of a sample is represented using a gray image with size of $1440 \times 1250$ pixels.

We also scanned $30 \times 20 \mathrm{~cm}^{2}$ of the surface of the test samples using the Metris LC50 laser following a standardized procedure, the scanning process takes around seven minutes per sample. Equivalent gray images of $1440 \times 1250$ pixels are built. For this, on a surface of $30 \times 17.28 \mathrm{~cm}^{2}$ we compute the median of points in cells of $0.24 \times 0.12 \mathrm{~mm}^{2}$. The depth vales are also converted into the rank from 1 to 256 values. Examples of the resulting images using both lasers on a particular sample are shown in Figure 3.

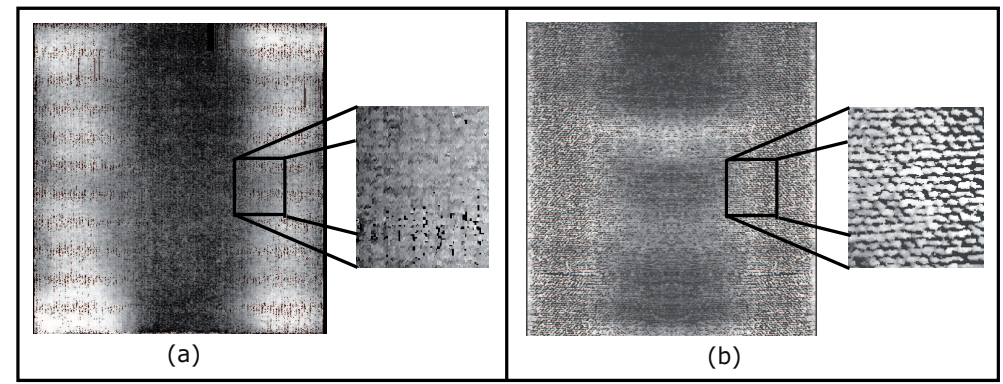

Figure 3: Sample acquired with a) the Metris LC50 laser and b) our proposed 3D scanner.

We evaluate the transitional change in texture from the original to the worn regions in the image. To do this, we independently evaluate the right and the left side. In both sides, we move a window, termed $M$, along the width a number of $W$ times to describe transitional changes of the texture. The degree of texture change is estimated comparing the texture from a transitional $M_{w}$ to the original $M_{1}$, with $w$ from 2 to $W$. A sub-window is moved $H$ times along the height of each moving window to increase the reliability of the estimation by using more texture samples. The moving window and the moving subwindows are shown in Figure 4 (a).

To evaluate the texture on an image, we extract features from each sub-window by using a rotational invariant version of the Local Binary Pattern technique named LBPROT. ${ }^{18}$ By this, the texture is described 
by evaluating the height relationship between a pixel and points on a circle around it. On the circle, equally spaced points are considered and the corresponding value is interpolated from the four closest pixels. Each point gets a code bit 0 or 1 assigned depending on whether it has a higher or lower grey value than the central pixel. These bits are read out clock wise and placed in a diadic code word named pattern. Rotated versions of patterns are grouped to make the description more compact and more robust to noise. To be able to use RBPROT, we resize the images to $720 \times 1250$ pixels to obtain pixels representing $0.24 \mathrm{~mm}^{2}$ of the sample surface. 12 equally distributed points are computed from a $5 \times 5$ pixels window around each pixel by using a radius of $\sqrt{2}$ (See Figure 4 (b)). Thus, 24 pixels are included in the calculation of each pattern, resulting in patterns very sensible to small texture variations.

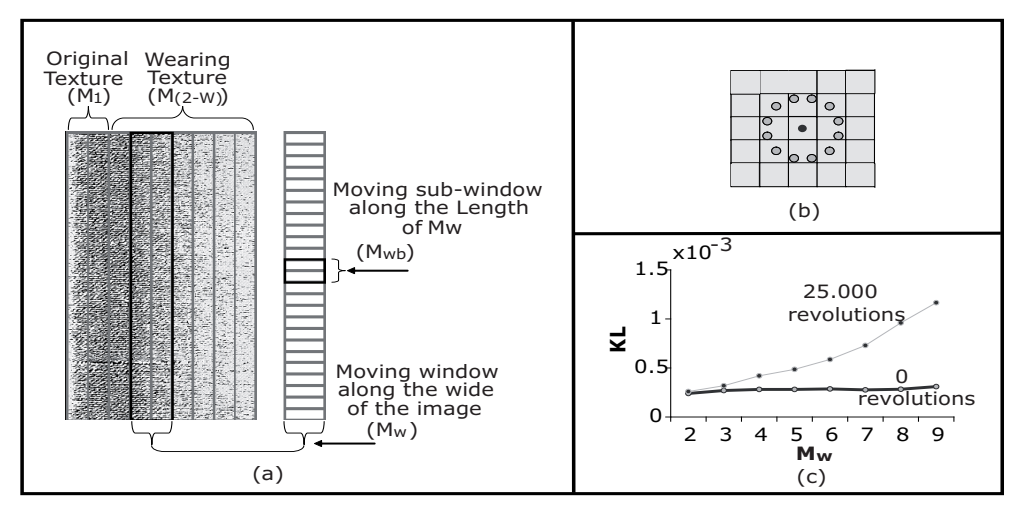

Figure 4: (a)Evaluation of the texture on the right side of an image. (b) Circular equally spaced points around a pixel in a $5 \times 5$ pixels windos. (c) KL values for two samples of loop pile.

To estimate the degree of change in texture on a particular $M_{w}$, we compare the difference between the distribution of the LBPROT patterns in $M_{w}$ and the distribution of the LBPROT patterns in $M_{1}$. The comparison is performed by using a symmetrized adaptation of the Kullback-Leibler divergence, termed KL. ${ }^{19,20}$ For this, we use $S$ pairs of sub-windows (one from $M_{1}$ and one $M_{w}$ ). We denote a given sub-window in $M_{1}$ by $a$ and a given sub-window in $M_{w}$ by $b$, with $a$ and $b$ from 1 to $H$, thus $S=H^{2}$ and $s=(a-1) H+b$ from 1 to $S$. The two corresponding LBPROT histograms of a given pair $\left(M_{1 a}, M_{w b}\right)$ are normalized into $h_{1 a}(i)$ and $h_{w b}(i), i$ is the bin index and $N$ is the total number of bins. Each bin corresponds to one LBPROT pattern. The KL divergence between sub-windows $W_{1 a}$ and $W_{w b}$ is computed as follows:

$$
(K L)_{s}^{w}=\sum_{i=1}^{N} h_{1 a}(i) \log h_{1 a}(i)+\sum_{i=1}^{N} h_{w b}(i) \log h_{w b}(i)-\sum_{i=1}^{N}\left(h_{1 a}(i)+h_{w b}(i)\right) \log \left(h_{1 a}(i)+h_{w b}(i)\right)+2 \log 2 .
$$

Evaluating a particular $M_{w}$, several factors such as flecks or speckles in the surface that could not be removed by the vacuum cleaning can cause KL values to be larger than any of the others. These outliers are detected using the Mahalanobis distance ${ }^{21}$ and then replaced with the median. The degree of change in texture of $M_{w}$, termed $C_{w}$ is then estimated using the mean of the KL values.

$$
C_{w}=\frac{1}{S} \sum_{s=1}^{S}(K L)_{s}^{w}
$$

Example of the $C_{w}$ values for test samples subjected to 0 and 25000 revolutions on the vettermas drum are shown in Figure 4 (c). The figure shows KL values monotonically increasing with the degree of wear for test sample $E$ and constant KL values for test sample $O$. This is a good indication that the degree of wear on a worn sample can be estimated by computing KL values and by comparing the texture in a region with 
original texture to a region with worn texture. From this finding, we propose to measure the degree of change in texture on the test samples, computing for each one a number of $S$ KL values, comparing $M_{1}$ to $M_{W}$. The degree of texture change of a test sample $t$ is defined as $C_{t}$. To compare the performance of both scanners, we check the degree of monotonicity and the linearity between $C_{t}$ and $t$. For this, we compute:

1. $\rho$ as the Spearman rank correlation coefficient. ${ }^{22}$ To compute $\rho$, the $C_{t}$ values are first ordered from small to large. Then, the difference between the assigned rank and the expected rank is computed as $d_{t}$ for each $C_{t}$ value. The significance is finally assessed by computing Eq. 3, with $K=6$, being a constant established by Spearman.

$$
\rho=1-K \sum_{t=1}^{T} d_{t}^{2} /\left((T)\left((T)^{2}-1\right)\right)
$$

2. $r$ as the Pearson product-moment correlation coefficient. ${ }^{23} r$ is computed from the mean of the products of the standard scores of $C_{t}$ and $t$ as shown in Eq. 4, with $\bar{C}_{t}$ and $\bar{t}$ the corresponding means of the variables, $\sigma_{C}$ and $\sigma_{t}$ the standard deviations and $\left(C_{t}-\bar{C}\right) / \sigma_{C}$ and $(t-\bar{t}) / \sigma_{t}$ the standard scores.

$$
r=\frac{1}{T-1} \sum_{t=1}^{T}\left(\frac{C_{t}-\bar{C}}{\sigma_{C}}\right)\left(\frac{t-\bar{t}}{\sigma_{t}}\right)
$$

\section{RESULTS}

We first present the results for the three type of loop surfaces used as test sets, each containing $T=6$ textile floor covering samples, one with original texture appearance and five worn samples. Textures of the test samples were acquired with both scanners and then converted to images representing the depth. Two images were obtained per sample corresponding to right and left sides of the textile floor covering sample. On both right and left images, we used 40 sub-moving windows, each one with size of $200 \times 200$ pixels. $H=20$ submoving windows were used on the side with original texture ( $a$ from 1 to $H$ ) on a window $\left(M_{1}\right)$ with size of $200 \times 1250$ pixels. The other $H=20$ sub-moving windows were used on the opposite side containing the worn texture ( $b$ from 1 to $H$ ) on a window $\left(M_{2}\right)$ with same size. Thus, we compute $400 \mathrm{KL}$ values comparing $M_{1 a}$ to $M_{2 b}$ per test sample.

The Spearman rank correlation and the Pearson product-moment correlation coefficients were computed between the degree of texture change of the test samples, $C_{t}(t=1$ to 6$)$, and the test sample numbers $t$. The results are shown in Table 1.

Table 1: Comparison of the degree of monotonicity and the linearity between the degree of texture change and the number of revolutions per test set for both scanners.

\begin{tabular}{|c|c|c|c|}
\hline Scanner & $\begin{array}{c}\text { Test } \\
\text { Sample }\end{array}$ & $r$ & $\rho$ \\
\hline Scanner & Loop 1 & 0.819 & 0.942 \\
\cline { 2 - 4 } based on & Loop 2 & 0.882 & 0.942 \\
\cline { 2 - 4 } Structured Light & Loop 3 & 0.994 & 1.000 \\
\hline Metris & Loop 1 & 0.782 & 0.771 \\
\cline { 2 - 4 } $\begin{array}{c}\text { LC50 } \\
\text { Scanner }\end{array}$ & Loop 2 & 0.848 & 0.928 \\
\cline { 2 - 4 } & Loop 3 & 0.977 & 1.000 \\
\hline
\end{tabular}

An analysis of variance ${ }^{24}$ of the Spearman's rank correlations using the type of loop surfaces as blocks shows significant differences ( $p$-values $<0.1)$ at $90 \%$ of confidence between the performance of both scanners. A similar analysis of variance of the Pearson's linear correlations shows significant differences $(p$-values $<0.01)$ at $99 \%$ of confidence between the performance of both scanners. Therefore, Our scanner shows to capture best the degree of texture change of the surface of the textile floor covering samples. Results for detected differences using the Scheeffe test ${ }^{25}$ on both a)the Spearman's rank correlation and b)the Pearson's linear correlations are illustrated in Figure 5. 

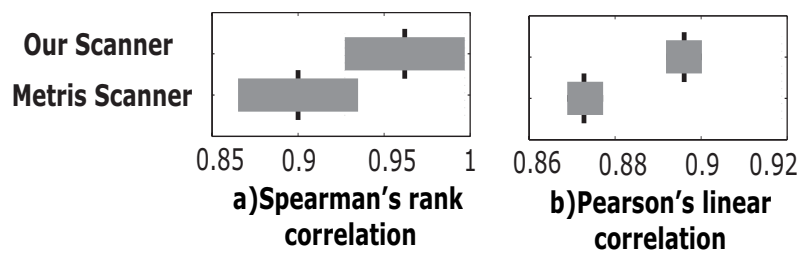

Figure 5: Both parameters, show the two lasers to be significantly different in performance and show our proposed scanner to perform better.

Secondly, we present the results of the Spearman rank correlation and the Pearson product-moment correlation coefficients computed between the degree of texture change of the reference samples, defined $C_{l}$, and the reference labels $l$. We use the same settings for $H, W, a, b$ and size of the images as these used for the test samples. The results are shown in Table 2 .

Table 2: Comparison of the degree of monotonicity and the linearity between the degree of texture change and the quality label for the reference samples.

\begin{tabular}{|c|c|c|c|}
\hline Scanner & $\begin{array}{c}\text { Reference } \\
\text { Sample }\end{array}$ & $r$ & $\rho$ \\
\hline \multirow{3}{*}{ Proposed } & Shaggy & 0.940 & 0.976 \\
\cline { 2 - 4 } & Frise & 0.943 & 0.976 \\
\cline { 2 - 4 } $\begin{array}{c}\text { Scanner } \\
\text { Based on } \\
\text { structured light }\end{array}$ & Shaggy & 0.950 & 0.976 \\
\cline { 2 - 4 } & Loop & 0.977 & 1.000 \\
\cline { 2 - 4 } & Cut loop & 0.959 & 0.952 \\
\cline { 2 - 4 } & High/Low loop & 0.850 & 0.779 \\
\cline { 2 - 4 } & Structured loop & 0.994 & 1.000 \\
\cline { 2 - 4 } & Woven cut pile & 0.878 & 0.809 \\
\hline
\end{tabular}

\section{DISCUSSION}

A comparison of proposed 3D scanner to a Metris LC50 3D scanner previously used by Waegeman ${ }^{13}$ has been conducted. Using features based on LBP statistics, we obtained for both scanners linear correlations above 0.78 and rank correlations above 0.77 between estimated degrees of wear and the number of revolutions applied with the Vetterman drum. An statistical analysis has shown that our 3D scanner performs better than the Metris scanner increasing an averange of 0.06 in the rank correlations and 0.03 in the linear correlation of the studied test samples. Our scanner is also $8.4(420 s / 50 s)$ times faster than the Metris laser scanner. Additionaly, a Metris LC50 laser scanner cost more than ten thousand Euro while our proposed scanner can be built with less than two thousand.

Waegeman has remarked that in previous attempts for automated assessment of carpet wear labels, few reference sets have been considered owing to great differences between textile floor covering types, resulting in a limited assessment performed successfully. Using the LBP statistics on different types of textile floor coverings including light and dark samples, we were able to obtain linear correlations above 0.88 and rank correlations above 0.77 . These results are a good indicator that further research using the depth images acquired with our proposed 3D scanner will yield to to a percentage of correct assessment of wear labels on carpets sufficiently enough for practical applications.

\section{CONCLUSSION}

In this paper we have presented a novel 3D scanner to capture the texture characteristics of worn carpets in depth images. We computed LBP statistics combined with an adaptation of the Kullback-Leibler divergence offering low computation cost estimating the degree of wear on textile floor covering samples. We measured two parameters: (1) the Spearman rank correlation and (2) the Pearson's linear correlation to evaluate the behavior of the extracted KL data compared to the number of revolutions of the Vetterman drum. We compared our proposed scanner to the Metris scanner using these parameters, scanning three types of loop pile surface of texture floor coverings with light colors. Both parameters, show the two lasers to be significantly different in 
performance and show our proposed scanner to perform better. Afterwards, we scanned the surface of samples from the standard EN1471 using our proposed scanner. We used also Spearman's and Pearson's correlations to evaluate the behavior of the KL data compared to the quality labels asigned by experts. The results show high correlations for both parameters on the different types of textile floor coverings. As a main conclusion, our proposed scanner offers additional benefits because it has been specifically designed for carpets, performing faster, cheaper, better and also a lot more suitable for darker carpets. The result of this approach gives optimistic expectations in the automation of the label assessment dealing with multiple types of carpets.

\section{ACKNOWLEDGMENTS}

The authors would like to thank the Department of Textiles in Ghent University, especially Didier Van Daele, for providing us the necessary materials to develop this approach.

\section{REFERENCES}

[1] http://www . carpet-rug.org/commercial-customers/selecting-the-right-carpet/ quality-and-performance/retention-rating-scales.cfm, Consulted Jan (2010)

[2] CRI Test Method - 101, Technical Bulletin, The Carpet and Rug Institute, http://www . carpet-rug.org/ technical_bulletins/0307_CRI_TM_101.pdf Consulted Jan (2010).

[3] Carpet and Rug Institute, http://www.carpet-rug.org/, Consulted Jan (2010).

[4] Siew, L. H. Hodgson, R. and M. Wood, E. J., Texture Measures for Carpet Wear Assessment, IEEE Transactions on Pattern Analysis and Machine Intelligence, V. 10 , Issue 1, 92-105 (1988).

[5] Wood, E., Hofgson, R., Carpet Texture Measurement Using Image Analysis, Textile Research Journal, V.59, 1-12 (1989)

[6] Wu, Y. Pourdeyhimi, B. and Spivak, S.M. Texture Evaluation of Carpets Using Image Analysis, Textile Research Journal, V.61, 407 - 419 Jul (1991).

[7] Sobus, J. Pourdeyhimi, B. Gerde, J. and Ulcay, Y., Assessing Changes in Texture Periodicity Due to Appearance Loss in Carpets: Gray Level Co-occurrence Analysis, Textile Research Journal, V. 61, 557-567 Oct (1991).

[8] Xu, B. Assessing carpet appearance retention by image analysis, Textile Research Journal. V.64, 497-509 (1994).

[9] Wang, J. and Wood, E.J., A New Method for Measuring Carpet Texture Change, Textile Research Journal, V.64, 215-224 Apr (1994).

[10] Sette, S. Boullart, L. Kiekens, and P., Self-Organizing Neural Nets: A New Approach to Quality in Textiles, Textile Research Journal, V.65, 196-202 Apr (1995).

[11] Van Steenlandt, W. Collet, D. Sette, S. Bernarn, P. Luning, R. Teze, L. Bohland, H. and Schulz, H., Automatic assesment of Carpet Wear Using Image Analysis and Neural Networks, Textile Research Journal, V.66, 55-561 (1996).

[12] The Deutsche Teppich-Forschungsinstitut, http://www.tfi-online.de/

[13] Waegeman, W. Cottyn, J. Wyns, B. Boullart, L. De Baets, B. Van Langenhove L. and Detand, J., Classifying Carpets Based on Laser Scanner data, Engineering Applications of Artificial Intelligence, V. 21, Issue 6, 907-918 Sep (2008).

[14] Textile Department, http://textiles.ugent.be/docs/RD/Carpets.pdf

[15] Orjuela, S. A. Copot, C. Syafiee, S. Vansteenkiste, E. De Keyser, R. Van Langenhove L. and Philips, W., Carpet Wear Classification Using Coocurrence Matrices and Support Vector Machines, XIII Simposio de Tratamiento de Senales, Imagenes y Vision Artificial (STSIVA 2008), ISBN: 978-958-8477-00-8, Sep (2008).

[16] ASTM D6119-05 Standard, http://www.astm.org/Standard/index.shtml, Consulted Jan (2010)

[17] European Committee for standarization, EN 1471:1996/A1, Textile floor coverings-Assessment of Changes in Appearance, European Committee for standarization, October (2003).

[18] Ojala, T. Pietikainen, M. and Menp, T. Multiresolution Gray Scale and Rotation Invariant Texture Classification with Local Binary Patterns, Computer Vision, ECCV 2000 Proceedings, Lecture Notes in Computer Science, Springer, 404-420 (2000). 
[19] Petrou, M. and Sevilla, P. G., Image Processing Dealing with texture, Wiley, ISBN: 9780470026281, (2006).

[20] Kullback, S. Leibler, R.A., On Information and Sufficiency, Annals of Mathematical Statistics 22. 79-86 (1951).

[21] Mahalanobis, P. C., On the Generalised Distance in Statistics, Proc. National Institute of Sciences of India 4955. 11-05 (1936).

[22] Spearman, C., The Proof and Measurement of Association Between Two Things, American Journal of Psycholy 15, 72-101 (1904).

[23] Fisher, R. A., Frequency Distribution of the Values of the Correlation Coefficient in Samples from an Indefinitely Large Population, Biometrika 10, 507-521 (1915).

[24] Fisher, R. A., Statistical Methods for Research Workers, 5th Edition., (2005).

[25] Milliken, G. A. and Johnson, D. E., Analysis of Messy Data, CRC Press, ISBN 0412990814, 35-36 (1993). 